\title{
Aspects about using Onicescu method for studying problems of environmental quality
}

\author{
Luminita GRECU ${ }^{1}$, a \\ ${ }^{1}$ University of Craiova, A.I. Cuza Street, no. 13, Craiova, Dolj, Romania \\ alumigrecu@hotmail.com
}

Keywords: multi-criteria decision models, ranking, Onicescu method, air quality.

\begin{abstract}
The aim of the paper is to present some aspects about Onicescu multi-criteria decision method and its application in decision problems regarding environmental quality. The mentioned method is applied to an air pollution problem, namely a problem in which we want to rank the counties from a certain region with respect to a complex air quality characteristic, a characteristic which incorporates all the most important air pollutants that exist in the considered region. Both variants of this method are applied in this paper and a comparison study between them is also made.
\end{abstract}

\section{Introduction}

Sometimes it is quite hard to take a decision, to make a choice when, for a given problem, many solutions exist, when many criteria of choosing have to be used. In such situations, so, when consequences that can be measured by different kind of measure units exist, it is necessary to obtain a qualitative and a quantitative evaluation for the global satisfaction degree of all criteria, in fact, to find out what decision variant is preferred.

Different multi-criteria decision models are widely used for solving problems arising in different domains, and among them literature highlights the global utility method, TOPSIS method, AHP, ELECTRE methods, Onicescu method, and others, $[1,2,3,4,5]$. In case of problems regarding the environment, these methods can be useful tools for making good decisions regarding the environment quality, regarding investments, or establishing the efficiency of applied solutions. In the herein paper we apply one of these methods, namely Onicescu method, to a problem regarding air pollution to point out its simplicity and to highlight some of its features.

As everyone knows air pollution is one of the most important problems in protecting healthy environment of this world. Environmental assessment is in our days among the highest priorities in socio - economic development around the world.

Oltenia Region is interested in environmental protection because, as other regions in the world, it has air pollution problems due, especially, to different industrial activities. There are differences between its regions and sometimes it is important to establish in which of them the whole environment improves, or where and if the environment policies achieve their desired goals. This thing is hard to be done in the absence of a complex indicator for the air pollution.

There are many indicators used for environmental assessment, but, as regarding air pollution, each of them refers to a single atmospheric pollutant, and none of them can be used to make an overall judgment. For a complex analysis, so for considering the concurrent action of all the important pollutants one can use for example multi-criteria decision models.

In this paper we apply Onicescu method to the air pollution data from Oltenia region, in order to rank the regions with respect to all most important air pollution sources taken together, these sources being four air pollutants which appear mostly in any region. The study is made for the values of these pollutants during 2011 year. The data used are known from national and regional environmental reports. 


\section{Base steps in applying Onicescu method}

Onicescu method has two important variants: one of them can be applied when the criteria envisaged have the same importance and the other when their importance is different. In the following a brief description of its variants is made.

Onicescu method - first variant. We suppose of having $m$ variants of decision, $V_{i}, i=\overline{1, m}$ and $n$ criteria for doing the choice operation, $c_{j}, j=\overline{1, n}$. The main steps to be followed when applying this method are:

1. the matrix of consequences, noted $A=\left(a_{i j}\right)_{1 \leq i \leq m, 1 \leq j \leq n}$, must be first built; element $a_{i j}$ is the value which represents the satisfaction degree of $c_{j}$ criteria, in case of choosing variant, $V_{i}$, $i=\overline{1, m}$.

2. matrix $\mathrm{B}$ is obtained as follows: for each criterion involved the decisional variants are ranked according to the type of criterion- for a maximum criterion we make a descending ranking, and for a minimum criterion an ascending one;

3. a new matrix, noted $C=\left(c_{i j}\right)_{1 \leq i \leq m, 1 \leq j \leq n}$, is then obtained by numbering how many times each variant $\mathrm{i}$ occupies position $\mathrm{j}$;

4. the decisional variants are ranked according to Onicescu aggregation function:

$$
f:\left\{V_{1}, . ., V_{m}\right\}, f\left(V_{i}\right)=\sum_{k=1}^{m} c_{i k} \frac{1}{2^{k}} .
$$

The optimum variant is the one that occupies the first position in a descending hierarchy.

Onicescu method - second variant. The first two steps are identically with those of the first variant. For the last ones one has to proceed as following:

3. The coefficients of importance are established using the formula: $p_{j}=\frac{1}{2^{k}}$, where $k$ represents the position of criterion $c_{j}$ in the descending ranking according to their importance. For obtaining their descending hierarchy we can use many methods as: the direct comparison method, the entropy method, the matrix method, etc.

4. Variants are ranked according to the aggregation function:

$$
f:\left\{V_{1}, . ., V_{m}\right\}, f\left(V_{i}\right)=\sum_{k=1}^{n} p_{k} \cdot 2^{-\alpha_{i k}}
$$

where $\alpha_{i k}$ represents the place occupied by $V_{i}$ with respect to criterion $k$.

The optimum variant is the one that occupies the first position if arranging them in a descending order according to this function values.

\section{Modeling the problem of air quality as a multi-criteria decision problem}

Using Onicescu method a concrete problem related to the air quality in Oltenia region is solved. We are interested in establishing which county from this region is the worst, regarding air pollution, taking into account the most common pollutants frequently met in the atmosphere.

We first must establish which are the alternatives or variants, and how many do we have, which are the criteria, and what consequences each of the considered variants has. 
We establish as alternatives (variants) the counties of Oltenia region, considered in an alphabetical order, namely: Dolj, Gorj, Mehedinti, Olt and Valcea, noted $V_{1}, V_{2}, V_{3}, V_{4}, V_{5}$. The main air pollutants found in this region: carbon oxide $(\mathrm{CO})$, nitrogen dioxide $\left(\mathrm{NO}_{2}\right)$ sulfur dioxide $\left(\mathrm{SO}_{2}\right)$, and particulate matter (PM-10), are considered to be the criteria used to make the choice. So we have five variants $(m=5)$ and four criteria $(\mathrm{n}=4)$, denoted by: $c_{1}, \ldots, c_{4}$.

The matrix of consequences, $A$, with 5 rows and 4 columns, contains the values of the environmental indicators of the considered pollutants in each county, obtained from the environmental reports on 2011, see [6], which are presented in Table 1.

Table 1. Air pollutants emission (t/an)-year 2011

\begin{tabular}{|l|l|l|l|l|}
\hline & $c_{1}\left(\mathrm{CO}_{2}\right)$ & $c_{2}\left(\mathrm{NO}_{2}\right)$ & $c_{3}\left(\mathrm{SO}_{2}\right)$ & $c_{4}(\mathrm{PM}-10)$ \\
\hline$v_{1}$ & 5785 & 14792 & 104378 & 851 \\
\hline$v_{2}$ & 13632 & 32822 & 115665 & 4297 \\
\hline$v_{3}$ & 24533 & 2888 & 56204 & 1389 \\
\hline$v_{4}$ & 339 & 2934 & 2123 & 1379 \\
\hline$v_{5}$ & 2000 & 6270 & 20923 & 460 \\
\hline
\end{tabular}

So, after step one, $A$-the matrix of consequences-is built:

$$
A=\left(\begin{array}{cccc}
5785 & 14792 & 104378 & 851 \\
13632 & 32822 & 115665 & 4297 \\
24533 & 2888 & 56204 & 1389 \\
339 & 2934 & 2123 & 1379 \\
2000 & 6270 & 20923 & 460
\end{array}\right)
$$

Both variants of this method are applied in order to establish which is more suitable when dealing with multi-criteria decisions problems of environmental quality.

We fist apply Onicescu method in its first variant, so we consider that all criteria involved have the same importance. Pointing out the fact that we want to find out the worst situation, we want to choose the worst case-the county for which the air pollution is the highest- we consider the above criteria as being of type max because the higher the value of an environmental indicator the better is the rank of the row for that specific indicator.

At the second step we evaluate matrix $B$, ranking the variants according with the criteria involved. We get:

$$
B=\left(\begin{array}{llll}
v_{3} & v_{2} & v_{2} & v_{2} \\
v_{2} & v_{1} & v_{1} & v_{3} \\
v_{1} & v_{5} & v_{3} & v_{4} \\
v_{5} & v_{4} & v_{5} & v_{1} \\
v_{4} & v_{3} & v_{4} & v_{5}
\end{array}\right)
$$

During the following step matrix $C \in M_{m^{2}}(N)$ is built. Its elements represent how many times each variant occupies a certain place, namely $c_{i j}$ represents how many times variant $i$ occupies place $j$ in matrix $B, c_{i j} \in\{0,1, \ldots . . n\}$. Evaluating $C$ we get: 


$$
C=\left(\begin{array}{lllll}
0 & 2 & 1 & 1 & 0 \\
3 & 1 & 0 & 0 & 0 \\
1 & 1 & 1 & 0 & 1 \\
0 & 0 & 1 & 1 & 2 \\
0 & 0 & 1 & 2 & 1
\end{array}\right)
$$

At step 4 the variants hierarchy is made according to Onicescu aggregation function, $f$ given by (1). In table 2 the values of this function are presented.

Table 2. Values of Onicescu aggregation function-first variant

\begin{tabular}{|l|l|}
\hline variants & $f$-Onicescu I \\
\hline 1 & 0.6875 \\
\hline 2 & 1.75 \\
\hline 3 & 0.90625 \\
\hline 4 & 0.25 \\
\hline 5 & 0.28125 \\
\hline
\end{tabular}

A descending order of these values lead to the following ranking: $V_{2}, V_{3}, V_{1}, V_{5}, V_{4}$.

So, after applying first variant of Onicescu method, we can say that $V_{2}$ is the choice, so the worst situation regarding air pollution is found in Gorj County, followed by Mehedinti, Dolj, Valcea and Olt.

When applying the first variant of Onicescu method all criteria involved have the same importance but for applying the second variant we must establish a hierarchy for the criteria involved. This hierarchy is very important because it has a great impact on the coefficients of importance as it would be further shown, and so it influences the final result. When applying Onicescu method the influence is grater than when applying other methods taking into account the way these coefficients are evaluated.

In case of the following descending order of their importance: $c_{1}, c_{2}, c_{3}, c_{4}$, at the third step we get the following coefficients of importance, presented in table 3:

Table 3. Coefficients of importance

\begin{tabular}{|l|r|r|r|r|}
\hline criteria & \multicolumn{1}{l|}{$\mathrm{CO}$} & \multicolumn{1}{|l|}{$\mathrm{NO}_{2}$} & $\mathrm{SO}_{2}$ & \multicolumn{1}{l|}{$\mathrm{PM}$} \\
\hline rank & 1 & 2 & 3 & 4 \\
\hline $\mathrm{p}$ & 0.5 & 0.25 & 0.125 & 0.0625 \\
\hline
\end{tabular}

At step four the variants hierarchy is made according to the aggregation function given by relation (2). The values of this function are presented in table 4.

Table 4. Values of Onicescu aggregation function-second variant

\begin{tabular}{|r|r|}
\hline variants & \multicolumn{1}{|r|}{$f$-Onicescu II } \\
\hline 1 & 0.16015625 \\
\hline 2 & 0.34375 \\
\hline 3 & 0.2890625 \\
\hline 4 & 0.04296875 \\
\hline 5 & 0.07226563 \\
\hline
\end{tabular}

So, after applying the second variant of Onicescu method, we obtain in this case the same hierarchy: $V_{2}, V_{3}, V_{1}, V_{5}, V_{4}$ and so the conclusion is the same: the worst situation for 2011 
regarding the air pollution by taking into account the action of all the four main pollutants considered above, is found in Gorj County, followed by Mehedinti, Dolj, Valcea and Olt.

Many other different studies can be made using this method. For example if we want to rank the regions considering other descending order for the criteria involved it is possible to not find the same ranking/hierarchy, fact that shows the importance of establishing the correct order of criteria. All the pollutants we refer to in this paper have dangerous consequences regarding human health, but, if we are interested especially in one of them, we can use the second variant considering the corresponding criteria as being the most important in the hierarchy of criteria.

If we consider the following descending order of their importance: $c_{2}, c_{3}, c_{1}, c_{4},\left(\mathrm{NO}_{2}, \mathrm{SO}_{2}, \mathrm{CO}\right.$, PM-10) at the third step we get the coefficients of importance, presented in table 5.

Table 5. Coefficients of importance

\begin{tabular}{|l|r|r|r|r|}
\hline criteria & \multicolumn{1}{|l|}{$\mathrm{CO}$} & \multicolumn{1}{|l|}{$\mathrm{NO}_{2}$} & \multicolumn{1}{|l|}{$\mathrm{SO}_{2}$} & \multicolumn{1}{l|}{$\mathrm{PM}$} \\
\hline rank & 3 & 1 & 2 & 4 \\
\hline $\mathrm{p}$ & 0.125 & 0.5 & 0.25 & 0.0625 \\
\hline
\end{tabular}

The values of the aggregation function obtained in this case are presented in table 6 :

Table 6. Values of Onicescu aggregation function-second variant

\begin{tabular}{|r|l|}
\hline variants & $f$-Onicescu II \\
\hline 1 & 0.207031 \\
\hline 2 & 0.4375 \\
\hline 3 & 0.109375 \\
\hline 4 & 0.050781 \\
\hline 5 & 0.103516 \\
\hline
\end{tabular}

The variants hierarchy based on the above values is: $V_{2}, V_{1}, V_{3}, V_{5}, V_{4}$, so the worst situation is still found in Gorj County but the second position is now occupied by Dolj County, instead of Mehedinti which occupied this position in the previous situation. Now this county is situated on the third place. Nothing changes regarding the last two positions where we find the same order: Valcea followed by Olt.

This example shows that the final ranking depends on the considered hierarchy of the criteria envisaged.

From relation (2) we also observe that the coefficients of importance decrease really fast, especially when we have a great number of criteria. For example in case of ten criteria the coefficient of importance used in relation (2) for the lowest placed criterion will be $\frac{1}{2^{10}} \cong 0.0009766$, a very small number comparing with the coefficient used for the first placed criterion, namely 0.5 . This example shows that in such cases, the lower placed criteriacan become negligible in the final value of Onicescu function.

In such situations, when the number of criteria is quite big other methods as for example, the global utility method, TOPSIS method or ELECTRE method, can be used.

\section{Conclusions}

In this paper we have applied Onicescu method in order to rank Oltenia's regions with respect to all important air pollution sources taken together. After applying both variants of Onicescu method, we can say that $V_{2}$ is the choice, so the worst situation regarding air pollution in 2011 is found in Gorj County. 
Considering that the pollutants have the same importance in establishing this ranking we have applied the first variant of Onicescu method. As we have mentioned before Gorj County presents the worst situation followed by Mehedinti, Dolj, Valcea and Olt.

When the criteria involved don't present the same importance the second variant of Onicescu method has to be applied. The results depend not only on the characteristics of variants but also on the importance given to criteria involved. We have shown in the herein paper that the criteria importance has a great influence on the result by considering two situations. When a certain descending order between the criteria has been chosen, namely: $\mathrm{CO}, \mathrm{NO}_{2}, \mathrm{SO}_{2}, \mathrm{PM}-10$, we found the same result as in case of the first variant. When this order was changed the final result changed too. Even if the worst situation was found in Gorj County, the second position was occupied by Dolj County, not by Mehedinti County.

The results obtained with the first variant of Onicescu method, and with the second one, in case of considering the first order between the criteria involved, are in concordance with those obtained in [7] where other two multi-criteria decision methods were applied for the same problem, and of for the same hierarchy of criteria importance.

Taking into account the way the coefficients of importance are evaluated when applying Onicescu method we may conclude that the hierarchy of criteria influences the final results more than in case of applying any of the other methods (in any other case).

We also have to mention that in case of a great number of criteria, for not losing the influence of the low placed criteria in the preferences hierarchy, Onicescu method has to be avoided. In such cases other multi-criteria decision methods may be use.

The envisaged problem is only an example of how we can use multi-criteria decision techniques for studying problems regarding environment quality. Similar studies regarding air quality can be made in other regions, for different periods of time, or considering other criteria. Other kind of environmental problems can be studied too with the mentioned methods: problems for evaluating alternatives in an environmental impact study; problems for evaluating which pesticides should be monitored in priority in the atmosphere; problems for making a hierarchy of strategies to mitigate road air pollution; and examples could continue. But for each problem it is important to choose the right method, the right variants and criteria, and their relative importance.

\section{References}

[1] J. Figueira, Greco, S. and Ehrgott, M., Multiple Criteria Decision Analysis: State of the Art Surveys, Springer, New York, 2004.

[2] R. E. Steuer, Multiple Criteria Optimization: Theory, Computation and Application, Wiley, New York, 1986.

[3] E. Triantaphyllou, Multi-Criteria Decision Making Methods: A Comparative Study, Kluwer Academic Publishers, Dordrecht, 2000.

[4] Satinee Lertprapai, Montip Tiensuwan, An Application of Multiple Criteria Decision Making in Combining Environmental Indices of Five Air Pollution Indicators, Thailand Statistician, July 2009;7(2):131-145.

[5] I. Vaduva, On solving Some Types of Multiple Attribute Decision-Making Problems, Romanian Journal of Economic Forecasting, 1/2012: 41-61.

[6] Information on http://arpmdj.anpm.ro/Mediu/raport.....

[7] L. Grecu, Aspects about using multi-criteria decision models for making decisions regarding environmental quality and protection, International Conference "Advances in Engineering and Management", ADEM 2012, pag. 143-146. 